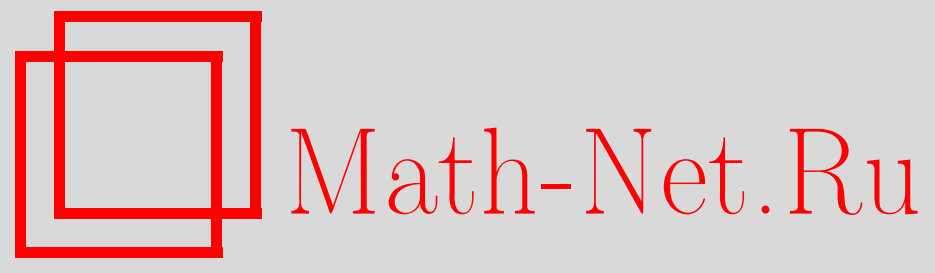

В. В. Стружанов, А. В. Коркин, А. Е. Чайкин, Об одном подходе к определению предельной несущей способности механических систем с разупрочняющимися элементами, Вестн. Сам. гос. техн. унта. Сер. Физ.-мат. науки, 2018, номер 4, 762-773

DOI: https://doi.org/10.14498/vsgtu1624

Использование Общероссийского математического портала MathNet.Ru подразумевает, что вы прочитали и согласны с пользовательским соглашением

http://www . mathnet.ru/rus/agreement

Параметры загрузки:

IP: 54.84 .234 .179

26 апреля 2023 г., 14:03:24

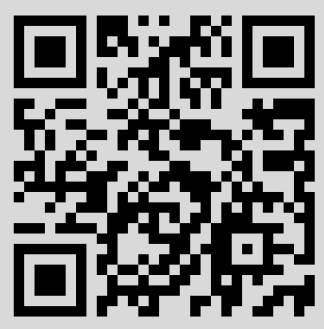


Вестн. Сам. гос. техн. ун-та. Сер. Физ.-мат. науки. 2018. Т. 22, № 4 . С. $762-773$ ISSN: 2310-7081 (online), 1991-8615 (print)

УДК 539.3

\title{
Об одном подходе к определению предельной несущей способности механических систем с разупрочняющимися элементами
}

\author{
В. В. Стружанов ${ }^{1}$, А. В. Коркин ${ }^{2}$, А. Е. Чайкин \\ 1 Институт машиноведения УрО РАН, \\ Россия, 620049, Екатеринбург, ул. Комсомольская, 34 \\ 2 Институт естественных наук и математики, \\ Уральский федеральный университет, \\ Россия, 620000, Екатеринбург, ул. Куйбышева, 48.
}

\begin{abstract}
Аннотация
Изложены основные положения теории расчета предельных нагрузок, действующих на дискретные механические системы с разупрочняющимися элементами. Методика опирается на численное определение вырожденных критических точек потенциальной функции системы, где происходит переход от устойчивости процесса нагружения к неустойчивости (катастрофа, разрушение), и позволяет избежать решения большого числа нелинейных уравнений равновесия. В качестве примера применения методики решена задача об определении предельного внутреннего давления в тонкостенном цилиндрическом резервуаре. При построении потенциальной функции системы использован специально построенный единый потенциал для плоского квадратного элемента материала в условиях двухосного растяжения, описывающий все стадии деформирования, включая и разупрочнение.
\end{abstract}

Ключевые слова: потенциальная функция, вырожденные критические точки, единый потенциал, матрица Гессе, тонкостенный резервуар, предельное давление.

Получение: 11 мая 2018 г. / Исправление: 11 октября 2018 г. / Принятие: 12 ноября 2018 г. / Публикация онлайн: 11 декабря 2018 г.

\section{Краткое сообщение}

( @) (7) Контент публикуется на условиях лицензии Creative Commons Attribution 4.0 International (https://creativecommons.org/licenses/by/4.0/deed.ru)

\section{Образец для цитирования}

Стружанов В. В., Коркин А. В., Чайкин А. Е. Об одном подходе к определению предельной несущей способности механических систем с разупрочняющимися элементами // Вестн. Сам. гос. техн. ун-та. Сер. Физ.-мат. науки, 2018. Т. 22, № 4. С. 762-773. doi: $10.14498 /$ vsgtu1624.

\section{Сведения об авторах}

Валерий Владимирович Стружанов (1) http://orcid.org/0000-0002-3669-2032 доктор физико-математических наук, профессор; главный научный сотрудник лаб. микромеханики материалов; e-mail: stru@imach.uran.ru

Александр Владимирович Коркин (D) http://orcid.org/0000-0003-3533-4257

аспирант; e-mail: alexkorkin@list.ru

Алексей Евгенъевич Чайкин (1) http://orcid.org/0000-0001-5582-2384

аспирант; e-mail: chaykin. ae@yandex.ru 
Введение. Вычисление предельной несущей способности механических систем основывается на расчете параметров равновесных состояний при постепенно возрастающей нагрузке. Затем эти параметры тем или иным способом сводят к некоторому скаляру и сравнивают его с предельной величиной, полученной в эксперименте. Например, на каждом шаге догружения определяют напряженное состояние элемента конструкции, находят соответствующий инвариант тензора напряжений, который сравнивают с его предельной величиной, взятой из эксперимента.

Возможен и другой подход, основанный на исследовании устойчивости положений равновесия, когда разрушение связывают с невозможностью равновесия, то есть с моментом потери устойчивости $[1,2]$. Реализация данного подхода возможна, если учитывать физически неустойчивые состояния материала (разупрочнение) [2-6]. Но и в этом случае возникает необходимость в решении не удовлетворяющих условиям корректности по Адамару [7] больших систем нелинейных уравнений равновесия для определения критических точек потенциальной функции механической системы, что является нетривиальной задачей [8]. Кроме того, выделение из них вырожденных критических точек, где происходит смена устойчивости на неустойчивость, требует применения нетрадиционного аппарата теории катастроф [9, 10]. Однако информация о параметрах равновесия (критических точках потенциальной функции системы) при возрастающих нагрузках, вообще говоря, является излишней. При определении предельной несущей способности нужно знать только вырожденные критические точки. В данной работе представлена методика, которая позволяет избежать аналитического решения нелинейных уравнений. Она основана на специальной числовой процедуре приближенного выбора вырожденных критических точек. В качестве примера рассмотрена задача о разрушении цилиндрического резервуара под действием внутреннего давления. Для построения потенциальной функции разработана методика построения единого потенциала для элемента материала, находящегося в плоском напряженном состоянии и описывающего его свойства как при упрочнении, так и при разупрочнении.

1. Общие положения. Положение механической системы в пространстве определяется конечным набором обобщенных координат (параметров состояния) $q_{i}, i=1,2, \ldots, N$. Величины параметров состояния зависят от набора задаваемых параметров управления системой $s_{j}, j=1,2, \ldots, M$ (внешние силы, перемещения некоторых точек системы, физико-механические свойства элементов и т. п.). Причем параметры состояния должны принимать такие значения, при которых механическая система находится в состоянии равновесия. Разрушение системы есть явление того же порядка, что и явление невозможности равновесия [1]. Когда равновесие становится неустойчивым, то реализуется или скачкообразный переход в ближайшее устойчивое равновесие, или глобальная катастрофа (разрушение). Поэтому под предельными значениями параметров управления следует понимать такие их величины, при реализации которых нарушается устойчивость системы.

В тех случаях, когда механическая система является градиентной [10], для определения предельных значений параметров управления можно воспользоваться следующей методикой. Градиентная система при квазистатическом нагружении с постоянной температурой описывается потенциальной функци- 
ей (лагранжианом) П $\left(q_{i}, s_{j}\right)$, связывающей параметры состояния и управления. Эта функция есть сумма потенциальных функций элементов системы, которые могут быть как вогнутыми, так и выпукло-вогнутыми. Выпуклые вниз (вогнутые) участки отвечают устойчивым состояниям элемента, выпуклые (выпуклые вверх) - неустойчивым. Точки перехода от выпуклости к вогнутости - пограничные точки, являющиеся в общем случае седловыми точками соответствующих потенциальных функций.

Когда механическая система находится в равновесии (устойчивом или неустойчивом), для связи параметров состояния и управления в этом равновесии можно использовать уравнение Лагранжа второго рода [11]. Тогда уравнение равновесия имеет вид

$$
\frac{\partial \Pi\left(q_{i}, s_{j}\right)}{\partial q_{i}}=0, \quad i=1,2, \ldots, N .
$$

Если функция является выпукло-вогнутой, то при заданных управляющих параметрах система (1) может иметь одно или более одного решения, как устойчивых, так и неустойчивых. Следовательно, в данном случае не выполняются условия Адамара [7].

Все решения системы (1) для всевозможных параметров управления образуют совокупность критических точек потенциальной функции П. Известно [10], что смена типа равновесия (устойчивого или неустойчивого) происходит в вырожденных критических точках, которые определяются из совместного решения уравнений (1) и уравнения, получающегося приравниванием к нулю детерминанта матрицы устойчивости (матрицы Гессе потенциальной функции):

$$
\operatorname{det}\left|\frac{\partial^{2} \Pi\left(q_{i}, s_{j}\right)}{\partial q_{m} \partial s_{n}}\right|=0, \quad m, n=1,2, \ldots, N .
$$

Очевидно, что уравнение (2) выделяет из множества критических точек вырожденные критические точки.

Таким образом, для отыскания предельных значений параметров управления, при достижении которых система теряет устойчивость, сначала, как правило, вычисляются все критические точки (решения уравнений (1)), затем выделяются вырожденные точки и после подстановки их в уравнение (1) определяются соответствующие параметры управления. Те параметры управления, при которых в первый раз достигается вырожденная критическая точка, то есть происходит смена устойчивого состояния механической системы на неустойчивое, и будут предельными значениями внешних нагрузок.

При реализации данной системы нахождения предельных значений параметров управления необходимо искать решения большого числа нелинейных уравнений, что представляет достаточно сложную задачу. Однако задача о вычислении предельных значений параметров управления не требует, вообще говоря, знания всех критических точек функции П (параметров всех положений равновесия механической системы). Необходимы только вырожденные точки, удовлетворяющие уравнению (2). Кроме того, для технических приложений можно использовать их приближенные значения, вычисленные с достаточной степенью точности.

Вычисление приближенных значений вырожденных критических точек можно осуществить, применяя следующую численную процедуру. Рассмот- 
рим евклидово пространство $\mathbb{R}^{M} \times \mathbb{R}^{N}$ - декартово произведение евклидовых пространств размерности $M$ и $N$. Элементами этого пространства являются числа $\left(q_{i}, s_{j}\right)$. Задавая физически обоснованные пределы изменения параметров состояния и управления, получаем $(M+N)$-мерный куб в пространстве $\mathbb{R}^{M} \times \mathbb{R}^{N}$, который разбиваем сеткой узлов на множество «кубиков» с заданным шагом. Затем в каждом узле сетки разбиения вычисляем значения компонент матрицы Гессе и величину ее определителя. Таким образом, каждому узлу ставится в соответствие значение детерминанта матрицы Гессе.

После этого выделяем те узлы, в которых детерминант близок к нулю с достаточной степенью точности. Полученное множество $B$ точек пространства $\mathbb{R}^{M} \times \mathbb{R}^{N}$ содержит в себе все вырожденные критические точки потенциальной функции П. Чтобы выделить из множества $B$ критические точки функции П, следует подставить элементы множества $B$ в уравнения равновесия (1). Те из них, которые удовлетворяют этим уравнениям с заданной степенью точности, будут вырожденными критическими точками. Параметры управления, соответствующие ближайшей (по евкледовой метрике) к началу координат вырожденной точке, образуют совокупность искомых предельных значений управлений. Если существует группа вырожденных точек, находящихся на одинаковом наименьшем расстоянии от начала координат, то получим различные сочетания значений параметров управления.

Таким образом, минуя стадию расчета параметров равновесных состояний, можно найти критические нагрузки, что достаточно для оценки прочности механических систем.

2. Модельный пример. Применим изложенную выше методику к определению предельных параметров управления (нагрузок) при растяжении модельной стержневой системы, изображенной на рис. 1. Стержень 2- абсолютно упругий с жесткостью при растяжении, равной $c$. Стержень 1 изготовлен из разупрочняющегося материала.

2.1. При мягком нагружении растягивающая сила $p$ - параметр управления, а удлинение $x$ стержня 1 и перемещение $u$ правого свободного конца стержня 2-параметры состояния (см. рис. 1). Сопротивление растяжению стержня 1 задано функцией $g(x)$ (зависимость растягивающей силы от удлинения), обладающей как восходящей ветвью (упрочнение), так и ветвью, падающей до нуля (стадия разупрочнения) [2,5]. В этом случае его потенциальная функция, равная $\int_{0}^{x} g(x) d x$, является выпукло-вогнутой, а лагранжиан системы [5]

$$
Q^{p}=\int_{0}^{x} g(x) d x+\frac{c}{2}(u-x)^{2}-\int_{0}^{u} p d u
$$

где второе слагаемое - энергия упругих деформаций стержня 2, последнее-

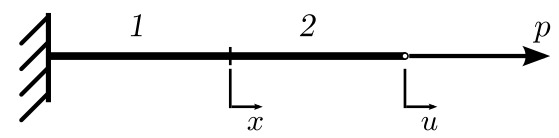

Рис. 1. Растягиваемая стержневая система с разупрочняющимся элементом

[Figure 1. A rod system with a softening element under tension] 
работа внешней силы, взятая со знаком минус.

Уравнения равновесия имеют вид

$$
Q^{p}{ }_{x}=g(x)-c(u-x)=0, \quad Q^{p}{ }_{u}=c(u-x)-p=0 .
$$

Здесь запятой обозначены частные производные по соответствующим аргументам. Приравнивая к нулю детерминант матрицы Гессе, получим уравнение

$$
g,{ }_{x}=0 .
$$

Отметим, что это уравнение определяет максимум функции $g(x)$. Его можно разрешить, используя следующую процедуру.

Проведем разбиение оси $X$ узлами с достаточно малым шагом. Подставляя координаты этих узлов в уравнение (4), находим среди них значение $x^{\prime}$, приближенно ему удовлетворяющее. Затем из уравнений (3) для данного $x^{\prime}$ определяем $p^{\prime}$ - предельную величину растягивающей силы.

2.2. В случае жесткого нагружения, когда растяжение осуществляется заданным перемещением $u$ (параметр управления), а $x$ является единственным параметром состояния, лагранжиан системы есть функция $Q^{u}$, равная двум первым слагаемым в выражении для $Q^{p}$.

Тогда имеем лишь одно уравнение равновесия (первое уравнение в системе (3)). В матрице Гессе только один элемент. Тогда равенство детерминанта нулю дает уравнение $g_{x}^{\prime}+c=0$. Его приближенное решение ищем так же, как и решение уравнения (4).

В зависимости от величины параметра $c$ возможны три варианта.

В первом - решения не существует. Следовательно, деформирование системы идет равновесно вплоть до разделения стержня 1 на части при значении $x=x^{z}$, когда падающая ветвь функции $g(x)$ достигает оси $X$. Предельное значение параметра управления получаем из уравнения равновесия после подстановки $x=x^{z}$ :

$$
u^{z}=g\left(x^{z}\right) / c+x^{z}
$$

Во втором варианте имеется только одно приближенное решение $x=x_{1}$. После достижения параметром $x$ значения этой величины система переходит в неустойчивое состояние. Поэтому предельный параметр управления

$$
u_{1}=g\left(x_{1}\right) / c+x_{1} \text {. }
$$

И, наконец, в третьем варианте имеем два решения $x_{1}, x_{2}\left(x_{1}>x_{2}\right)$. Тогда предельное значение $u=u_{1}$.

Отметим, что такие же результаты были получены и в работах $[5,8]$ только после решения нелинейных уравнений равновесия при постепенном возрастании параметров управления и построения соответствующих многообразий катастроф и сепаратрис, состоящих из вырожденных критических точек.

3. Вогнуто-выпуклый потенциал при двухосном растяжении тонкой пластины. Для реализации положений теории, изложенной выше, необходимо знать вогнуто-выпуклый потенциал элементов системы. В механике сплошной среды эта функция должна быть определена для элемента материала вплоть до разупрочнения и разделения на фрагменты. 
Рассмотрим плоский квадратный элемент материала с единичными размерами, находящийся в плоско-напряженном состоянии, причем касательные напряжения и деформации отсутствуют. Деформирование осуществляется заданием нормальных деформаций $\varepsilon_{1}, \varepsilon_{2}$ (двуосное растяжение). Если материал находится в состоянии упругости, то его потенциальная энергия

$$
V^{e}\left(\varepsilon_{1}, \varepsilon_{2}\right)=\frac{1}{2}\left(\sigma_{1} \varepsilon_{1}+\sigma_{2} \varepsilon_{2}\right)=\frac{E}{2\left(1-\nu^{2}\right)}\left(\varepsilon_{1}^{2}+2 \nu \varepsilon_{1} \varepsilon_{2}+\varepsilon_{2}^{2}\right),
$$

где $E$ - модуль Юнга, $\nu$ - коэффициент Пуассона. Здесь использован закон Гука, связывающий нормальные напряжения $\sigma_{1}, \sigma_{2}$ и нормальные деформации $\varepsilon_{1}, \varepsilon_{2}$ и записанный в векторно-матричной форме:

$$
\left(\begin{array}{l}
\sigma_{1} \\
\sigma_{2}
\end{array}\right)=C\left(\begin{array}{l}
\varepsilon_{1} \\
\varepsilon_{2}
\end{array}\right)
$$

В этом выражении

$$
C=\frac{E}{\left(1-\nu^{2}\right)}\left(\begin{array}{cc}
1 & \nu \\
\nu & 1
\end{array}\right)
$$

можно трактовать как матричный коэффициент пропорциональности.

Формула (5) определяет кривые второго порядка на плоскости $\varepsilon_{1} \varepsilon_{2}$ (линии уровня потенциала $\left.V^{e}\right)$, а именно центральные эллипсы, главные оси которых наклонены к декартовым осям $\varepsilon_{1}, \varepsilon_{2}$ под углом $\pi / 4$. Большая ось располагается во втором и четвертом квадрантах, малая - в первом и третьем. Для полуосей выполняется отношение

$$
\frac{a^{2}}{\rho^{2}}=\frac{(1-\nu)}{(1+\nu)}
$$

где $a$ - малая полуось, $\rho$ - большая полуось. Точки, расположенные на малой оси, отвечают равномерному растяжению $\left(\varepsilon_{1}=\varepsilon_{2}\right)$ или сжатию $\left(-\varepsilon_{1}=-\varepsilon_{2}\right)$, а точки на большой оси определяют чистый сдвиг $\left(\varepsilon_{1}=-\varepsilon_{2},-\varepsilon_{1}=\varepsilon_{2}\right)$. Произвольная точка $\left(\varepsilon_{1}, \varepsilon_{2}\right)$ лежит на линии уровня с большой полуосью, равной

$$
\rho=\frac{1}{\sqrt{2}} \sqrt{\left(\varepsilon_{1}+\varepsilon_{2}\right)^{2} \frac{1+\nu}{1-\nu}+\left(\varepsilon_{1}-\varepsilon_{2}\right)^{2}} .
$$

При чистом сдвиге $\left(\varepsilon_{1}=\varepsilon, \varepsilon_{2}=-\varepsilon\right) \rho=\gamma / \sqrt{2}$, где $\gamma=\varepsilon_{1}-\varepsilon_{2}=2 \varepsilon-$ сдвиг. После исчерпания упругости для описания свойств материала можно было бы использовать теорию малых упруго-пластических деформаций $[12,13]$, в которой предполагается упругость объемной деформации и пропорциональность девиаторов тензоров напряжений и деформаций. Однако после выхода на стадию разупрочнения первая гипотеза не выполняется, так как материал уже существенно поврежден множеством микротрещин и объемный модуль изменяется.

Чтобы остаться в рамках деформационной теории, следует отказаться от первой гипотезы, а вместо второй сохранить пропорциональность напряжений и деформаций с некоторым уже переменным, зависящим от деформаций 
матричным коэффициентом. Например, в рассматриваемой задаче этот матричный коэффициент в законе (6) можно принять в виде $C \psi\left(\varepsilon_{1}, \varepsilon_{2}\right)$, где $\psi-$ некоторая скалярная функция, требующая специального определения.

Следствием принятого предположения является тот факт, что в области неупругости линии уровня функции энергии деформаций $V$ (потенциальной энергии) подобны линиям уровня потенциала $V^{e}$, то есть представляют собой эллипсы с тем же самым отношением главных осей. Необходимо только знать распределение значений потенциала по этим линиям уровня.

Воспользуемся полной диаграммой для чистого сдвига $\tau(\gamma)(\tau$ - касательное напряжение, $\gamma$ - деформация сдвига), которая обладает восходящей и нисходящей до нуля ветвями. Такую диаграмму материала можно получить, используя опытную диаграмму кручения стержней круглого поперечного сечения с пересчетом на диаграмму материала [14]. Тогда энергия, затраченная на деформирование, есть

$$
V=\int_{0}^{\gamma} \tau(\gamma) d \gamma
$$

После взятия интеграла и замены $\gamma$ на $\sqrt{2} \rho$ с учетом выражения (7) получаем искомый единый потенциал $V\left(\varepsilon_{1}, \varepsilon_{2}\right)$.

Пусть $\tau=0.1 G \gamma(1-5 \gamma)$, если $0 \leqslant \gamma \leqslant 0.2$, где $G=\frac{E}{2(1+\nu)}$ - модуль сдвига в упругости. Тогда

$$
V\left(\varepsilon_{1}, \varepsilon_{2}\right)=\left\{\begin{array}{cc}
\frac{V^{e}}{2}\left(0.1-\frac{1}{3} \sqrt{\frac{V^{e}}{G}}\right), & \left(\varepsilon_{1}, \varepsilon_{2}\right) \in \Omega, \\
0.002 G / 3, & \left(\varepsilon_{1}, \varepsilon_{2}\right) \notin \Omega .
\end{array}\right.
$$

Очевидно, что область $\Omega$ ограничена эллипсом с полуосями $\rho=0.141$; $a=\rho \sqrt{(1+\nu) /(1-\nu)}$. Качественный вид поверхности $V\left(\varepsilon_{1}, \varepsilon_{2}\right)$ изображен на рис. 2. Функция $V$ описывает все состояния материала (выпуклость вниз устойчивость, упрочнение; выпуклость вверх - неустойчивость, разупрочнение).

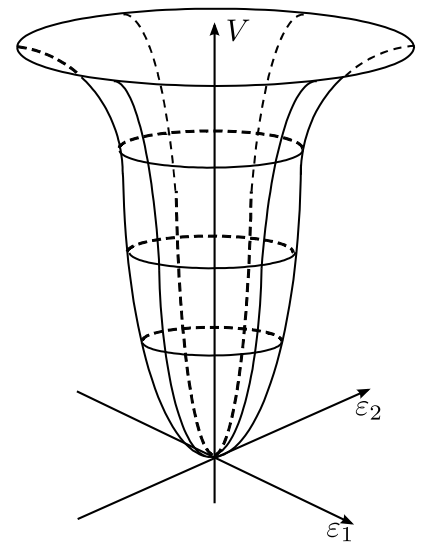

Рис. 2. Качественный вид поверхности потенциала $V\left(\varepsilon_{1}, \varepsilon_{2}\right)$

[Figure 2. Qualitative form of the potential surface $\left.V\left(\varepsilon_{1}, \varepsilon_{2}\right)\right]$
Связь между напряжениями и деформациями (определяющие соотношения) в данном случае будет задана законом

$$
\left(\begin{array}{c}
\sigma_{1} \\
\sigma_{2}
\end{array}\right)=\left(\begin{array}{l}
\frac{\partial V}{\partial \varepsilon_{1}} \\
\frac{\partial V}{\partial \varepsilon_{2}}
\end{array}\right)=C \psi\left(\varepsilon_{1}, \varepsilon_{2}\right)\left(\begin{array}{l}
\varepsilon_{1} \\
\varepsilon_{2}
\end{array}\right)
$$

где

$$
\psi\left(\varepsilon_{1}, \varepsilon_{2}\right)=\frac{1}{2}\left(0.1-\frac{1}{2} \sqrt{\frac{V^{e}}{G}}\right) .
$$

Отметим, что напряжения являются компонентами градиента функции $V$. 
4. Разрушение цилиндрического резервуара. Рассмотрим тонкостенный резервуар в виде длинной трубы с заделанными абсолютно жесткими крышками торцами. Радиус трубы равен $b$, длина $l$, толщина стенки $t(t \ll b)$. Резервуар находится под действием монотонно и квазистатически возрастающего при постоянной температуре внутреннего давления с интенсивностью $p$ $[15,16]$. Материал обладает свойством деформационного разупрочнения. Применим изложенную выше методику для расчета предельного значения давления.

Каждый элемент трубы находится в плоском напряженном состоянии при всестороннем растяжении: $\varepsilon_{1}=\varepsilon_{z}-$ продольная деформация, $\varepsilon_{2}=\varepsilon_{\theta}$ - тангенциальная деформация. Его потенциальная функция $V$ задана выражениem (8).

Механическая система (резервуар) обладает двумя параметрами состояния (обобщенными координатами) $\varepsilon_{z}, \varepsilon_{\theta}$ (однородное напряженно-деформированное состояние) и одним параметром управления $p$.

Лагранжиан (потенциальная функция) этой системы

$$
W=V\left(\varepsilon_{z}, \varepsilon_{\theta}\right) 2 \pi b l t-p \pi b^{2} l \varepsilon_{z}-2 \pi p b^{2} l \varepsilon_{\theta} .
$$

Здесь первое слагаемое - полная энергия деформаций стенок трубы, второе и третье - взятые со знаком минус соответственно работа сил давления на перемещении жестких крышек и перемещении, равном увеличению радиуса. Тогда уравнения равновесия, определяющие критические точки функции $W$, имеют вид

$$
\frac{\partial W}{\partial \varepsilon_{z}}=\frac{\partial V}{\partial \varepsilon_{z}} 2 \pi b l t-p \pi b^{2} l=0 ; \quad \frac{\partial W}{\partial \varepsilon_{\theta}}=\frac{\partial V}{\partial \varepsilon_{\theta}} 2 \pi b l t-2 p \pi b^{2} l=0 .
$$

Уравнения (9) сводятся к одному:

$$
2 \frac{\partial V}{\partial \varepsilon_{z}}=\frac{\partial V}{\partial \varepsilon_{\theta}}
$$

Производя необходимые вычисления, находим, что

$$
\varepsilon_{\theta}=\frac{2-\nu}{1-2 \nu} \varepsilon_{z}
$$

то есть в пространстве деформаций путь деформирования элемента материала является прямой линией, уравнение которой задается равенством (10). Кроме этого, из уравнений (9) следует, что $\sigma_{z}=p b / 2 t, \sigma_{\theta}=p b / t$. Отсюда путь нагружения в пространстве напряжений также является прямой линией. Таким образом, имеем ситуацию с пропорциональным нагружением элемента материала.

Определим, наконец, предельное значение внутреннего давления. Как следует из приведенных выше теоретических положений, для этого необходимо гессиан функции $W$ приравнять к нулю, найти все решения полученного уравнения, по крайней мере, приближенно, и выделить из них вырожденные критические точки потенциала $W$, которые принадлежат множеству критических точек (решений уравнений (9)) потенциала. Имеем

$$
\left\{\frac{\partial^{2} V}{\partial \varepsilon_{z}^{2}} \cdot \frac{\partial^{2} V}{\partial \varepsilon_{\theta}^{2}}-\left(\frac{\partial^{2} V}{\partial \varepsilon_{z} \partial \varepsilon_{\theta}}\right)\right\} 4 \pi^{2} b^{2} l^{2} t=0
$$


После несложных преобразований получаем

$$
\begin{aligned}
& \left(0.05-\alpha \beta-\alpha \beta^{-1}\left(\varepsilon_{z}+\nu \varepsilon_{\theta}\right)^{2}\right)\left(0.05-\alpha \beta-\alpha \beta^{-1}\left(\varepsilon_{\theta}+\nu \varepsilon_{z}\right)^{2}\right)- \\
& -\nu^{2}\left(0.05-\alpha \beta-\alpha \beta^{-1}\left(\varepsilon_{z}+\nu \varepsilon_{\theta}\right)\left(\varepsilon_{\theta}+\nu \varepsilon_{z}\right)\right)^{2}=0
\end{aligned}
$$

где $\alpha=0.25(1-\nu)^{-1 / 2}, \beta=\left(\varepsilon_{z}^{2}+2 \nu \varepsilon_{z} \varepsilon_{\theta}+\varepsilon_{\theta}^{2}\right)^{1 / 2} ; \varepsilon_{z}, \varepsilon_{\theta} \geqslant 0,\left(\varepsilon_{z}, \varepsilon_{\theta}\right) \in \Omega$.

Для вычисления вырожденных критических точек функции $W$ применим следующую численную процедуру.

Построим на плоскости $\varepsilon_{z} \varepsilon_{\theta}$ квадрат $\Omega_{1}$ такой, что в нем $\varepsilon_{z}, \varepsilon_{\theta}>0$, $\left(\varepsilon_{z}, \varepsilon_{\theta}\right) \in \Omega \subset \Omega_{1}$ (рис. 3). Для определения стороны этого квадрата найдем на осях $\varepsilon_{\theta}$ и $\varepsilon_{z}$ равные отрезки, внутри которых детерминант матрицы Гессе меняет знак. Получаем отрезки длиной 0.1 . Эту величину и берем за сторону квадрата $\Omega_{1}$. Отметим, что данный прием может быть использован при построении $M \times N$-мерного куба в общей теории.

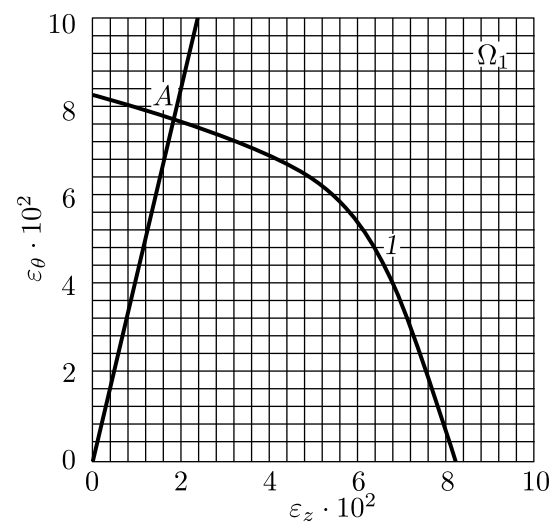

Рис. 3. Вид кривой, в точках которой гессиан равен нулю

[Figure 3. The curve where the Hessian is equal to zero at all points]

Разобьем этот квадрат прямоугольной сеткой с достаточно малым шагом (на рис. 3 показана укрупненная сетка). В каждом узле сетки по формуле (11) при $\nu=0.3$ вычисляем детерминант (гессиан) матрицы Гессе. Выделяем те из них, где детерминант мало отличен от нуля, что определяется заданной точностью вычислений.

Производя расчеты, получаем множество точек, расположенных на кривой 1 (рис. 3). Затем координаты точек кривой 1 подставляем в уравнения (9) и выделяем те из них, которые удовлетворяют уравнениям с заданной точностью. В результате находим только одну точку (точка $A$, рис. 3 ). Точка $A$ лежит на прямой (10) и имеет координаты $\varepsilon_{z}=0.018, \varepsilon_{\theta}=0.076$ (с точностью до округления). Это и есть искомая вырожденная критическая точка потенциальной функции $W$. Подставляя ее координаты в уравнения равновесия, находим критическое внутреннее давление, при котором происходит потеря устойчивости системы (катастрофа, разрушение). Если $E=2 \cdot 10^{5} \mathrm{MПа,} b=100 \mathrm{мм}, t=2 \mathrm{мм}$, то критическое (предельное) значение давления $p=18$ МПа. Заметим, что величина предельного давления увеличивается пропорционально толщине стенки. 
Конкурирующие интересы. Мы не имеем конкурирующих интересов.

Авторский вклад и ответственность. Все авторы принимали участие в разработке концепции статьи и в написании рукописи. Все авторы несут полную ответственность за представление окончательной рукописи в печать. Окончательная версия рукописи была одобрена всеми авторами.

Финансирование. Исследование не имело финансирования.

\section{Библиографический список}

1. Седов Л. И. Механика сплошной среды. Т. 1. М.: Наука, 1970. 492 с.

2. Стружанов В. В., Миронов В. И. Деформачионное разупрочнение материала в элеменmax конструкций. Екатеринбург: УрО РАН, 1995. 190 с.

3. Вильдеман В. Э., Чаусов Н. Г. Условия деформационного разупрочнения материала при растяжении образца специальной конфигурации // Заводская лаборатория. Диагностика материалов, 2007. Т. 73, № 10. С. 55-59.

4. Вильдеман В. Э., Третьяков М. П. Испытания материалов с построением полных диаграмм деформирования // Проблемы машиностроения и надежности машин, 2013. № 2. С. 93-98.

5. Стружанов В. В., Коркин А. В. Об устойчивости процесса растяжения одной стержневой системы с разупрочняющимися элементами // Вестник Уральского государственного университета путей сообщения, 2016. №3(31). С. 4-17. doi:10.20291/ 2079-0392-2016-3-4-17.

6. Андреева Е. А. Решение одномерных задач пластичности для разупрочняющегося материала // Вестн. Сам. гос. техн. ун-та. Сер. Физ.-мат. науки, 2008. №2(17). С. 152160. doi: 10.14498/vsgtu642.

7. Арсенин В. Я. Методы математической физики и специальные функции. М.: Наука, 1974. $431 \mathrm{c}$.

8. Стружанов В. В., Коркин А. В. О решении нелинейных уравнений равновесия одной растягиваемой стержневой системы с разупрочняющимися элементами методом простых итераций // Вестник Уральского государственного университета путей сообщения, 2017. № 2(34). C. 4-16. doi : 10.20291/2079-0392-2017-2-4-16.

9. Poston T., Stewart I. Catastrophe Theory and Its Applications / Surveys and Reference Works in Mathematics. vol.2. London, San Francisco, Melbourne: Pitman, 1978. xviii +491 pp.

10. Gilmore R. Catastrophe theory for scientists and engineers / A Wiley-Interscience Publication. New York: John Wiley \& Sons, 1981. xvii+666 pp.

11. Pars L. A. A treatise on analytical dynamics. London: Heinemann Educational Books, 1965. $\mathrm{xxi}+641 \mathrm{pp}$.

12. Ильюшин А. А. Об основах общей математической теории пластичности // Вестник Московского университета. Серия 1. Математика. Механика, 1961. № 3. С. 31-36.

13. Качанов Л. М. Основы теории пластичности. М.: Наука, 1969. 420 с.

14. Стружанов В. В. Определение диаграммы деформирования материала с падающей ветвью по диаграмме кручения цилиндрического образца // Сиб. журн. индустр. матем., 2012. Т. 15, № 1. С. 138-144.

15. Радченко В. П. Введение в механику деформируемых систем. Самара: СамГТУ, 2009. 241 c.

16. Southwell R. V. An introduction to the theory of elasticity. For engineers and physicists / Oxford Engineering Science Series. Oxford: Clarendon Press, 1936. ix +510 pp. 


\title{
One approach to determination of the ultimate load-bearing capacity of mechanical systems with softening elements
}

\author{
V.V.Struzhanov ${ }^{1}$ A.V. Korkin ${ }^{2}$,A.E. Chaykin ${ }^{2}$ \\ 1 Institute of Engineering Science, Ural Branch of RAS, \\ 34, Komsomolskaya st., Ekaterinburg, 620049, Russian Federation. \\ 2 Institute of Natural Sciences and Mathematics, Ural Federal University, \\ 48, Kuybysheva, st., Ekaterinburg, 620000, Russian Federation.
}

\begin{abstract}
The fundamental provisions of the limiting load calculation theory are presented for a discrete mechanical system with softening elements. The method is based on the numerical determination of degenerate critical points for the potential function of the system. At these points there is a transition from the stability of the loading process to instability such as a catastrophe or a failure. This approach helps to avoid solving a large number of nonlinear equilibrium equations. The problem of determining the limiting internal pressure in a thin walled cylindrical tank is solved as an example. A unified potential specially defined for a flat square element of material in biaxial tension is used in developing a potential function of the system. It describes all stages of deformation including the softening stage.
\end{abstract}

Keywords: potential function, degenerate critical points, unified potential, Hesse matrix, thin walled reservoir, limiting pressure.

Received: $11^{\text {th }}$ May, $2018 /$ Revised: $11^{\text {th }}$ October, $2018 /$

Accepted: $12^{\text {th }}$ November, $2018 /$ First online: $11^{\text {th }}$ December, 2018

Competing interests. We have no competing interests.

Authors' contributions and responsibilities. Each authors has participated in the article concept development and in the manuscript writing. The authors are absolutely responsible for submitting the final manuscript in print. Each authors has approved the final version of manuscript.

\section{Short Communication}

○ ()(1) The content is published under the terms of the Creative Commons Attribution 4.0 International License (http://creativecommons.org/licenses/by/4.0/)

Please cite this article in press as:

Struzhanov V. V., Korkin A. V., Chaykin A. E. One approach to determination of the ultimate load-bearing capacity of mechanical systems with softening elements, Vestn. Samar. Gos. Tekhn. Univ., Ser. Fiz.-Mat. Nauki [J. Samara State Tech. Univ., Ser. Phys. Math. Sci.], 2018, vol. 22, no. 4, pp. 762-773. doi: 10.14498/vsgtu1624 (In Russian).

\section{Authors' Details:}

Valery V. Struzhanov (1) http://orcid.org/0000-0002-3669-2032

Dr. Phys. \& Math. Sci., Professor; Chief Reseacher; Lab. of Matherial Micromechanics; e-mail: stru@imach.uran.ru

Aleksandr V. Korkin (D) http://orcid.org/0000-0003-3533-4257

Postgraduate Student; e-mail: alexkorkin@list.ru

Aleksey E. Chaykin (10 http://orcid.org/0000-0001-5582-2384

Postgraduate Student; e-mail: chaykin.ae@yandex.ru 
Funding. This research received no specific grant from any funding agency in the public, commercial, or not-for-profit sectors.

\section{References}

1. Sedov L. I. Mekhanika sploshnoi sredy [Continuum Mechanics], vol. 1. Moscow, Nauka, 1970, 492 pp. (In Russian)

2. Struzhanov V. V., Mironov V. I. Deformatsionnoe razuprochnenie materiala $v$ elementakh konstruktsii [Deformational Softening of Material in Structural Elements]. Ekaterinburg, UrO RAN, 1995, 190 pp. (In Russian)

3. Vil'deman V. E., Chausov N. G. Conditions of strain softening upon stretching of the specimen of special configuration, Zavodskaia laboratoriia. Diagnostika materialov, 2007, vol. 73, no. 10, pp. 55-59 (In Russian).

4. Vil'deman V. E., Tretyakov M. P. Material testing by plotting total deformation curves, J. Mach. Manuf. Reliab., 2013, vol. 42, no. 2, pp. 166-170. doi: 10.3103/S1052618813010159.

5. Struzhanov V. V., Korkin A. V. Regarding stretching process stability of one bar system with softening elements, Vestnik Ural'skogo gosudarstvennogo universiteta putei soobshcheniia, 2016, no. 3(31), pp. 4-17 (In Russian). doi : 10.20291/2079-0392-2016-3-4-17.

6. Andreeva E. A. Solution of one-dimensional softening materials plasticity problems, Vestn. Samar. Gos. Tekhn. Univ., Ser. Fiz.-Mat. Nauki [J. Samara State Tech. Univ., Ser. Phys. Math. Sci.], 2008, no. 2(17), pp. 152-160 (In Russian). doi: 10.14498/vsgtu642.

7. Arsenin V. Ya. Metody matematicheskoi fiziki i spetsial'nye funktsii [Methods of mathematical physics and special functions]. Moscow, Nauka, 1974, 431 pp. (In Russian)

8. Struzhanov V. V., Korkin A. V. On the solution of non-linear equations of trim of a single expandable frame structure with softening elements by the method of simple iterations, Vestnik Ural'skogo gosudarstvennogo universiteta putei soobshcheniia, 2017, no. 2(34), pp. 416 (In Russian). doi : 10.20291/2079-0392-2017-2-4-16.

9. Poston T., Stewart I. Catastrophe Theory and Its Applications, Surveys and Reference Works in Mathematics, vol. 2. London, San Francisco, Melbourne, Pitman, 1978, xviii+491 pp.

10. Gilmore R. Catastrophe theory for scientists and engineers, A Wiley-Interscience Publication. New York, John Wiley \& Sons, 1981, xvii+666 pp.

11. Pars L. A. A treatise on analytical dynamics. London, Heinemann Educational Books, 1965, $\mathrm{xxi}+641 \mathrm{pp}$.

12. Ilyushin A. A. On the foundations of the general mathematical theory of plasticity, Vestn. Mosk. Univ., Ser. I, 1961, no. 3, pp. 31-36 (In Russian).

13. Kachanov L. M. Foundations of the theory of plasticity, North-Holland Series in Applied Mathematics and Mechanics, vol. 12. Amsterdam, North-Holland Publ., 1971, xiii+482 pp.

14. Struzhanov V. V. The determination of the deformation diagram of a material with a falling branch using the torsion diagram of a cylindrical sample, Sib. Zh. Ind. Mat., 2012, vol.15, no. 1, pp. 138-144.

15. Radchenko V. P. Vvedenie $v$ mekhaniku deformiruemykh sistem [Introduction to the mechanics of deformable systems]. Samara, Samara State Technical Univ., 2009, 241 pp. (In Russian)

16. Southwell R. V. An introduction to the theory of elasticity. For engineers and physicists, Oxford Engineering Science Series. Oxford, Clarendon Press, 1936, ix +510 pp. 